\title{
La enseñanza clínica, un reto de nuestro tiempo
}

\author{
Federico L Rodríguez Weber ${ }^{1}$
}

Gran verdad es decir que tiempos pasados no son iguales a los tiempos actuales y que el conocimiento y la trasmisión del mismo han tomado formas y mecanismos diferentes en cuanto a su uso, aplicación y aprendizaje. La medicina no escapa a este fenómeno. Dentro de la misma existen áreas que con el tiempo se han tornado de mayor importancia: la genética, la patología, el radiodiagnóstico... Éstas y otras, si bien siguen teniendo la misma importancia, han cambiado en su aplicación, como sucede con la propedéutica clínica, la patología clínica, la hematología y, por último, con otras más que en el proceso de formación son indispensables para la formación del médico, pero que han modificado su formato de aprendizaje, y posiblemente su importancia en el esquema curricular en relación con las generaciones pasadas, por ejemplo la histología, la anatomía y la bioquímica.

Otros grandes cambios, en comparación con hace 40 o 50 años, los encontramos en la incorporación de los recursos diagnósticos, los recursos terapéuticos, la profundidad del conocimiento de enfermedades, y lo mismo podríamos decir de la forma de enseñar los contenidos, las destrezas clínicas y la forma de relacionarse entre profesores-alumnos, alumnos-alumnos, profesorespacientes, pacientes-alumnos, y la interrelación de las diferentes áreas del conocimiento y el ejercicio médico, haciendo del proceso de enseñanza de la medicina más difícil y complicado por el número de variables y los recursos con los que se debe de contar para adquirir el conocimiento, así como para respetar las reglamentaciones vigentes.

\footnotetext{
1 Médico Internista. Profesor titular de introducción a la clínica. Profesor adjunto de la Residencia de Medicina Interna de la Universidad Mexicana de Medicina de la Universidad La Salle. Hospital Ángeles Pedregal.
}

Correspondencia:

Dr. Federico L Rodríguez Weber

Correo electrónico: fweber@saludangeles.com

Aceptado: 10-08-2016.

Este artículo puede ser consultado en versión completa en http:// www.medigraphic.com/actamedica
La tecnología, sin duda, ocupa un lugar importantísimo en el proceso de enseñanza de la medicina y hoy se tienen recursos a la mano que antes tan sólo eran anecdóticos, por ejemplo los maniquíes, los robots, los modelos inteligentes, el Internet o los sistemas digitales. Todos útiles para la enseñanza. La clínica no escapa a la tecnología ni a la simulación, pero ninguno de estos recursos sustituye al paciente real en situación real y en tiempo real, y donde el medio ambiente, la familia y los recursos propios de cada paciente y de cada consultorio u hospital, hacen de cada situación clínica un caso único que el estudiante tendrá que tomar en cuenta para enfrentar el problema en su proceso de aprendizaje, partiendo de que todo estudiante de medicina, tarde o temprano, tendrá que entrar en contacto con los pacientes, teniendo o no los recursos docentes modernos. El estar con el paciente y obtener la información del mismo para llegar a un diagnóstico mediante la aportación de la historia clínica, el interrogatorio y la exploración, y con esos mismos recursos evaluar el progreso en la mejoría o el empeoramiento, para así apoyar nuestras observaciones con los estudios pertinentes y confirmar la integración del conocimiento adquirido en las diferentes materias, hace de la enseñanza, y en especial de la clínica y su propedéutica, una materia de gran trascendencia en la formación del médico, además de confirmar que el contacto con los pacientes es insustituible. ${ }^{1,2}$

Por lo anterior, no podemos dejar de ver la realidad, pues para tener pacientes se requiere de campos clínicos, los cuales cada vez son más difíciles de obtener ya que son especialmente competidos; por un lado, con la aparición desmedida y no totalmente supervisada de escuelas de medicina. Los campos clínicos son muy solicitados por las universidades y la sobrepoblación de estudiantes hace que la oportunidad de adquirir experiencia con casos reales sólo sea parcial. Otro factor a tomar en cuenta es la disponibilidad de los pacientes, reticentes en algunos casos, para colaborar con alumnos en formación (estudiante, internado o residencia), al no entender el beneficio de este proceso, e impidiendo ver lo útil que representa contar con la atención del estudiante en cualquiera de sus niveles. El paciente se ve favorecido cuando el equipo de salud pone más atención en el estudio y mayor entusiasmo en el control del paciente, mejorando el diagnóstico y tratamiento, y haciéndolo más oportuno, actualizado y con mayor evidencia, siempre que esta práctica 
sea supervisada y realizada con el más alto profesionalismo y ética, y corresponda a las actividades de la profesión, tanto por parte del profesor como del alumno. De esta forma se facilita una mejor atención al paciente ${ }^{3}$ y se da al estudiante la oportunidad de generar conocimiento significativo que podrá aplicar el día de mañana en una mejor atención de futuros pacientes, ${ }^{4,5}$ dejando claro que el aprendizaje de la clínica y sus materias puede ser parcialmente adquirido en maniquíes y robots; incluso el ejercicio académico puede realizarse con modelos inteligentes o con simulación u otros recursos, pero si no se cuenta con pacientes reales en situaciones reales será solamente un aprendizaje teórico, carente del significado que sólo se puede transmitir con el aprendizaje práctico y complejo, y ante los propios pacientes.

Por lo antes expuesto, invito a las autoridades en educación y salud, tanto de las universidades como a las autoridades hospitalarias, a facilitar este aprendizaje. A los profesores de clínicas los invito a que cuiden sus campos haciendo ver a sus pacientes y alumnos la importancia de los médicos en formación, y la necesidad de estar enterados continuamente del proceso clínico en que se encuentran, pues la participación de los alumnos asegura una atención de calidad y seguridad al paciente en el enfrentamiento con la enfermedad, ya que es muy probable que los alumnos, en fases clínicas, pasen más tiempo con los pacientes que el propio médico.

Por último, invitamos a todos los médicos en formación que tienen la oportunidad de contar con campos clínicos a que aprovechen su presencia con los pacientes encontrando en cada uno un libro de conocimientos que les permita adquirir la experiencia necesaria para enfrentar los retos cotidianos de la profesión médica en el ambiente de su ejercicio, ayudando a una mejor atención de los pacientes ante su proceso de diagnóstico, tratamiento y recuperación. ${ }^{6}$ Recordemos que en nuestro sistema educativo el paso de los estudiantes de pregrado en la clínica puede representar el último contacto con los pacientes en un campo real, y los de postgrado deben recordar que en el trato con cada paciente se acompaña la enseñanza que no se habrá de repetir, y que será invaluable en la formación particular del especialista.

\section{REFERENCIAS}

1. Kassirer J. Teaching clinical reasoning: case based coached. Acad Med. 2010; 85: 1118-1124.

2. Jopling H. The principles of clinical examination. J Clin Exam. 2006; 1: 3-6.

3. Rodríguez WF. Disciplina y autoridad: elementos en la formación de médicos especialistas profesionales. Med Int Mex. 2016; 32 (1): 115-117.

4. Barnett K, Mercer SW, Norbury M, Watt G, Wyke S, Guthrie B. Epidemiology of multimorbidity and implications for health care, research and medical education: a cross-sectional study. The Lancent. 2012; 380 (9836): 37-43. doi: 10.1016/50140-6736.

5. Ruelas BE, Mansilla R. Las ciencias de la complejidad y la innovación médica. México. Plaza y Valdés. 2005.

6. Lifshitz GA. La enseñanza de la clínica en la era moderna. Onv Ed Med. 2012; 1 (4): 210-217. 